\title{
CATALOGUES OF THE FAINT OBJECTS IN THE AREAS WITH GAMMA-RAY BURSTS
}

\author{
V.V. Golovnia ${ }^{1}$, Yu.I. Protsyuk ${ }^{2}$, V.M. Andruk ${ }^{1}$, I.B. Vavilova ${ }^{1}$, L.K. Pakuliak ${ }^{1}$, \\ I.V. Kulyk ${ }^{1}$, Ya.O. Romanyuk ${ }^{1}$, O.R. Baransky ${ }^{3}$ \\ ${ }^{1}$ Main Astronomical Observatory NAS of Ukraine, Kyiv, Ukraine, \\ golov@mao.kiev.ua,andruk@mao.kiev.ua, \\ ${ }^{2}$ Nikolaev Astronomical Observatory, Mykolaiv, Ukraine,yuri@nao.nikolaev.ua \\ ${ }^{3}$ Taras Shevchenko National University of Kyiv, Kyiv, Ukraine
}

ABSTRACT. The results of GRB observations are being published in the GCN Circulars in real-time. To study all the objects in the sky areas around the GRBs we identified them on the digitized MAO Double Wide angle Astrograph (DWA) plates $(\mathrm{D} / \mathrm{F}=40 / 200)$ within a circle with the radius of dozens of arcminutes. We selected only GRBs with the positional accuracy between \pm 0.3 " and $\pm 7.5^{\mathrm{N}}$ and the range of magnitudes between $14^{\mathrm{m}}$ and $20^{\mathrm{m}}$.

We used MIDAS/ROMAFOT package to obtain the catalogues of the faint objects in small areas in the GRBs vicinity. For the plates of DWA telescope the positional rms errors are $\pm 0.20^{\prime \prime}$ for RA and DEC, the photometric errors are $\pm 0.20^{\mathrm{m}}$. All positions were obtained in Tycho- 2 system.

Keywords: gamma-ray burst, astronomical data base, methods: data analysis

\section{Introduction}

Gamma-ray bursts are the most powerful explosions known in the Universe. Now they are identified with the regions of formation of massive stars in galaxies and the parent short-lived explosions of massive stars, which have a random spatial distribution in the sky (Sokolov et al., 2009; Raikov et al., 2010; Kienlin.et al., 2014; Gerasim et al., 2015).

The astroplates give us an unique opportunity to identify the optical counterparts of the GRBs and study all the objects in the vicinity of the registered GRB (Golovnya et al., 2012, 2014; Pakuliak et al., 2013; Vavilova et al., 2014). With this aim we used MIDAS/ROMAFOT software package to obtain catalogues of the faint objects in small areas in the vicinity of the registered GRB. This software was developed at the MAO NAS of Ukraine for processing the scanned records to get a high precision astrometric and photometric data of objects (Andruk et al., 2005, 2007, 2010, 2014; Yatsenko et al., 2011; Golovnya et al., 2010).

\section{Search of optical analogs of GRB}

The results of GRB observations are being published in the GCN Circulars in real-time (Barthelmy, 2015). The Database of Joint Digital Archive (JDA) of Ukrainian Virtual Observatory (UkrVO), which contains observational data obtained at Ukrainian observatories, was used to search for optical analogs of GRBs on the astroplates (Sergeeva et al., 2004; Vavilova et al., 2010, 2012a, 2012; Pakuliak et al., 2012; Protsyuk et al., 2014).
Table 1 shows the optical coordinates of some GRBs and their precision (sigm) from GCN Circulars, for which the optical identifications were successful (Beardmore et al., 2015; Goad et al., 2015; Osborne et al., 2015; Evans et al., 2015). The table also lists numbers of the plates (N) from JDA UkrVO for the corresponding objects.

Table 1: The number of plates (N) in JDA UkrVO

\begin{tabular}{|c|c|c|c|}
\hline GRB & \begin{tabular}{l}
\multicolumn{3}{c}{ RA, Dec, J2000 } \\
$\mathrm{h}$ m s deg ' "
\end{tabular} & $\begin{array}{l}\text { Sigm } \\
", \pm\end{array}$ & $\mathrm{N}$ \\
\hline $150728 \mathrm{~A}$ & $19: 28: 55.28+33: 54: 57.8$ & 4.1 & 3 \\
\hline $150626 \mathrm{~B}$ & $12: 30: 32.04+66: 46: 18.6$ & 1.7 & 5 \\
\hline $150607 \mathrm{~A}$ & $09: 19: 57.25+68: 26: 09.6$ & 1.4 & 3 \\
\hline $150530 \mathrm{~A}$ & $21: 50: 02.94+57: 30: 59.8$ & 1.6 & 9 \\
\hline $150527 \mathrm{~A}$ & $19: 15: 50.32+04: 12: 06.9$ & 1.5 & 9 \\
\hline $150423 \mathrm{~A}$ & $14: 46: 18.96+12: 17: 00.6$ & 1.8 & 10 \\
\hline $150323 \mathrm{~A}$ & $08: 32: 42.74+45: 27: 52.8$ & 1.9 & 6 \\
\hline
\end{tabular}

However, the identification process was not always successful. Let us give an example of the search for GRB 140419A (Osborne et al., 2014). At the first detection this object had a magnitude of $\sim 13.5$, then it was increased to 12.5 in $\sim 70 \mathrm{sec}$ and then the brightness decayed fast to $\sim 14.3^{\mathrm{m}}$ for about $350 \mathrm{sec}$ (Guver et al., 2014). We found two plates obtained in 1978 and 1990 with the desired areas, but we could not find the optical analogue of this GRB. Also, no optical counterpart was found in the close vicinity of GRB 140818B (Beardmore et al., 2014). But inspecting larger area around this object, we found some images near GRB position on both selected plates. Brightness of the objects was estimated as $\mathrm{V}=15.0$ and $\mathrm{V}=16.5$ (Golovnya, 2014).

The five plates were found in JDA UkrVO for GRB $150212 \mathrm{~A}$ and GRB 150213B, but they had no optical analogue (Evans et al., 2015, Golovnya, 2015, Osborne et al., 2015, Golovnya, 2015).

Three plates with the GRB150220A (Evans et al., 2015) were found with a limited magnitude of $16.4,15.6$ and 15.7 respectively. Two objects were found on plate GUA040C002183, EPOCH=1993.2192. Fig. 1 shows $13^{\prime} \times 13^{\prime}$ area around GRB150220A with these two objects. The coordinates and $\mathrm{B}$ magnitudes were obtained in the system of TYCHO-2 catalogue with rms errors of 0.093", $0.099^{\prime \prime}, 0.21^{\mathrm{m}}$ for right ascension, declination, and magnitudes respectively (Golovnya \& Andruk, 2015). 


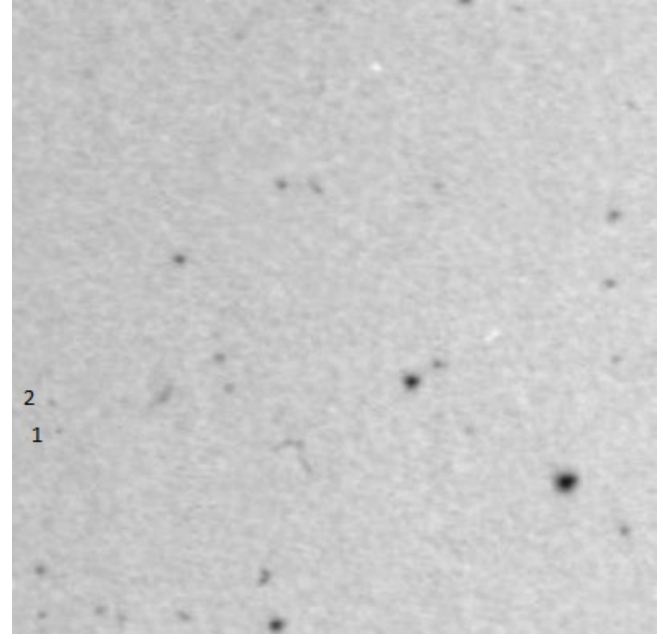

Figure 1: The area of GRB150220A with identified Obj1 and Obj2

We observed the same field around GRB 150220A with $0.7-\mathrm{m} \mathrm{f} / 4$ reflector of Kyiv comet station on Mar 10, 2015 during 18 days after the burst has been occurred. The telescope is equipped with CCD (1027x1056 pixels with a scale of $0.95 " / p x)$. We obtained $21 \mathrm{CCD}$ frames with 60 sec expositions in R-filter (Romanyuk et al., 2015). The individual frames were summed in order to increase the signal. We did not find aforementioned images of Obj1 and $\mathrm{Obj} 2$ on the final CCD frame. However, we confirmed some of the positions of X-ray sources reported early by P.A. Evans. Table 2 contains the coordinates of X-ray sources given by Evans et al. (2015) and their optical identifications made with the CCD.

Table 2: Source's position as follows by P.A.Evans (GCN 17501) and Ya.O.Romanyuk (GCN 17645).

\begin{tabular}{|c|c|c|c|c|}
\hline & \multicolumn{2}{|c|}{ GCN 17501} & \multicolumn{2}{|c|}{ GCN 17645} \\
\hline & \multicolumn{2}{|c|}{$\mathrm{h} m \mathrm{~s} \quad \circ{ }^{\prime}$} & \multirow{2}{*}{\multicolumn{2}{|c|}{$\begin{array}{c}\text { h m s } \\
\text { outside our field }\end{array}$}} \\
\hline 1 & 90131.65 & -013907.6 & & \\
\hline 2 & 90130.74 & -013827.0 & \multicolumn{2}{|c|}{ outside our field } \\
\hline 3 & 90208.55 & -013659.1 & 90208.1 & -013635 \\
\hline 4 & 90208.90 & -014020.1 & \multicolumn{2}{|c|}{ extremely faint } \\
\hline 5 & 90128.27 & -013906.3 & \multicolumn{2}{|c|}{ outside our field } \\
\hline 6 & 90225.29 & -014114.9 & 90225.3 & -014115 \\
\hline 7 & 90146.37 & -013813.9 & 90146.4 & -013814 \\
\hline
\end{tabular}

We detected the optical counterpart $\left(18.3^{\mathrm{m}}\right)$ of the GRB 151027A with 0.5-m telescope Mobitel KT-50 of the Nikolaev observatory with Alta-U9000 CCD with R-filter (Protsyuk \& Kovalchuk, 2015).

\section{Creation of catalogs of objects in the areas around GRBs}

As it was shown above, the optical counterparts of some GRBs and X-ray sources can be identified with the plates from the JDA UkrVO. With this aim we decided to create the catalogues of faint stars in the vicinity of GRBs (the catalogues are under development). At the first step we scanned the plates with resolution of $1200 \mathrm{dpi}$, but in order to increase a positional accuracy, we shall rescan the plates with resolution of $1600 \mathrm{dpi}$.
Special tools were also developed, which allowed obtaining the positions and magnitudes of faint stars with accuracy of about \pm 0.20 " and $\pm 0.20^{\mathrm{m}}$ respectively. Each catalogue covers the sky area of about 10-20 arcmin and contains the coordinates and magnitudes of about hundreds of stars. The TYCHO2 or UCAC4 catalogues are used as reference ones. The developed tools allow us the fast cross-identification between the lists of the obtained optical positions of the faint stars and GRB coordinates (Protsyuk et al., 2014a, 2014). The catalogues will be put on the UkrVO web-site after completing.

Acknowledgements. This work was partially supported by the Ukrainian Astronomical Association.

\section{References}

Andruk V.N. et al.: 2005, Kinematika i Fizika Nebesnykh Tel, 21, N5, 396.

Andruk V. et al.: 2007, J. Phys. Studies, 11, No3, 329.

Andruk V.M. et al.: 2010, Kinem. Physics Celest. Bodies, 26, No3, 146.

Andruk V.N. et al.: 2014, Odessa Astron. Publ., 27/1, 53.

Barthelmy S.: 2015, gcn.gsfc.nasa.gov.

Beardmore A.P. et al.: 2014, GCN 16712.

Beardmore A.P. et al.: 2015, GCN 18088.

Evans P.A. et al.: 2015, GCN 17879, GCN 17735.

Evans P.A. et al.: 2015, GCN 17452.

Evans P.A.: 2015, GCN 17501.

Gerasim R.V. et al.: 2015, Astrophys., 58/2, 204.

Goad M.R. et al.: 2015, GCN 17615.

Goad M.R. et al.: 2015, GCN 17968, GCN 17889.

Golovnya V. et al.: 2010, J. Phys. Studies, 14, No 2, 2902.

Golovnya V. et al.: 2012, Kyiv Univ. Messenger. Astronomy, 49, 36 (in ukrainian).

Golovnya V.V.: 2014, GCN 16757.

Golovnya V. et al.: 2014, www.astroplate.cz/wp-content/ uploads/2014/01/Golovnya_UkrVO_new_life.pdf

Golovnya V.V.: 2015, GCN 17473.

Golovnya V.V.: 2015, GCN 17484.

Golovnya V.V., Andruk V.M.: 2015, GCN 17589.

Guver T. et al.: 2014, GCN 16120.

Kienlin A. et al.: 2014, Astroph. J. Suppl. S., 211, 13.

Osborne J.P. et al.: 2014, GCN 16124.

Osborne J.P. et al.: 2015, GCN 17475.

Osborne J.P. et al.: 2015, GCN 17905.

Pakuliak L et al.: 2012, IAUS, 285, 389.

Pakuliak L. et al.: 2013, Odessa Astron. Publ., 26/2, 236.

Protsyuk Yu., Kovalchuk O.: 2015, GCN 18533.

Protsyuk Yu.I. et al.: 2014, Odessa Astron. Publ., 27/1, 59.

Protsyuk Yu.I. et al.: 2014, Kinemat. Physics Celest. Bodies, 30, N6, 54.

Raikov A.A. et al.: 2010, Astrophys., 53, 3, p.396.

Romanyuk Ya. et al.: 2015, GCN 17645.

Sergeeva et al.: 2004, Baltic Astronomy, 13, No 4, 677.

Sokolov V.V. et al.: 2009, Workshop "Many faces of GRB phenomena - optics vs high energy".

Vavilova I.B., Pakuliak L.K., Protsyuk Yu.I.: 2010, Kosmichna Nauka i Tekhnologiya, 16, 62.

Vavilova I.B. et al.: 2012, Kinem. Physics Celest. Bodies, 28, No2, 85.

Vavilova I.B. et al.: 2012, Baltic Astronomy, 21, 356.

Vavilova I. et al.: 2014, Odessa Astron. Publ., 27/1, 65.

Yatsenko A.I. et al.: 2011, Kinem. Physics Celest. Bodies, 27, No5, 249. 